\title{
Closed-Loop Estimation for Randomly Sampled Measurements in Target Tracking System
}

\author{
Jin Xue-bo, Lian Xiao-feng, Su Ting-li, Shi Yan, and Miao Bei-bei \\ College of Computer and Information Engineering, Beijing Technology and Business University, Beijing 100048, China \\ Correspondence should be addressed to Jin Xue-bo; xuebojin@gmail.com
}

Received 24 October 2013; Revised 30 December 2013; Accepted 30 December 2013; Published 26 February 2014

Academic Editor: Hamid Reza Karimi

Copyright (C) 2014 Jin Xue-bo et al. This is an open access article distributed under the Creative Commons Attribution License, which permits unrestricted use, distribution, and reproduction in any medium, provided the original work is properly cited.

\begin{abstract}
Many tracking applications need to deal with the randomly sampled measurements, for which the traditional recursive estimation method may fail. Moreover, getting the accurate dynamic model of the target becomes more difficult. Therefore, it is necessary to update the dynamic model with the real-time information of the tracking system. This paper provides a solution for the target tracking system with randomly sampling measurement. Here, the irregular sampling interval is transformed to a time-varying parameter by calculating the matrix exponential, and the dynamic parameter is estimated by the online estimated state with YuleWalker method, which is called the closed-loop estimation. The convergence condition of the closed-loop estimation is proved. Simulations and experiments show that the closed-loop estimation method can obtain good estimation performance, even with very high irregular rate of sampling interval, and the developed model has a strong advantage for the long trajectory tracking comparing the other models.
\end{abstract}

\section{Introduction}

Target tracking is the most important preliminary step for many higher-level analysis applications. Nowadays, some new sensors have been used in the tracking systems, such as the radio frequency identification (RFID) readers. The RFID stores and retrieves data through the electromagnetic transmission to an RF compatible integrated circuit. Once the tag gets close to the readers, the distance between the readers and tags can be got and sent to the data processing center. The measurements of RFID are randomly sampled [1] because of the data-driven measurement mechanisms. Datadriven approach has been used in many applications [2], and the irregular sampling is one of the important issues in this approach.

In general, the video tracking system has to extract the visual information at each frame [3], which costs much computing amount. In [4], the target is tracked by some selected frames to reduce the calculation cost and achieve the real-time tracking, which also results in the randomly sampled tracking problem. If the output measurements are obtained at a set of irregular sampling times, the traditional recursive estimation from $K$ to $K+1$ may fail in general [5]. Both the model and the estimation method should be reconsidered.

Reference [6] transformed the randomly sampled measurement tracking to some time-varying parameters and used the current model to describe the processing model [7-11], which assumes a priori probability density of the acceleration as Rayleigh density. Due to the randomly sampled measurement, this assumption is no longer satisfied.

Except the current model, there were several other models used in the tracking, such as constant-velocity (CV) model, constant acceleration model (CA), and Singer model (zero mean first-order Markov model) [12, 13]. The CV models [1] emphasize that the accelerations are small. In maneuvering target tracking, the inclusion of acceleration in the state vector would degrade tracking performance. The main attractive feature of this model is its simplicity. It is sometimes used in the maneuvering target tracking techniques, such as the so-called noise-level adjustment, when the maneuver is quite small or random. It is also simply referred to as the CA model or more precisely the nearly CA model. The Singer model regards the target acceleration 
as a first-order semi-Markov process with zero mean, which is in essence a priori model since it does not use online information on the target maneuver. Again because of the irregular sampling time, the priori model does not meet the actual dynamic model of the target.

The approach to update the system model online has attracted great interest of the researchers. For example, the interacting multiple model (IMM) [14, 15] method considers the change of the system dynamics as a Markovian parameter, whose transition probability is set based on the online estimation and then fusions several models for the tracking, while IMM suffers heavy computational burden on condition that the maneuvering target has complex motion. Moreover, the complex movement can also lead to frequent switch between different models, which can cause the tracking performance to decline. Another model $[16,17]$ estimated the state of a power system, where the bus voltages are transformed to a system parameter. But the works of this closed-loop estimation have not yet been involved in the randomly sampled tracking system.

This paper will develop a joint state-and-parameter estimation method for the target tracking system with randomly sampled measurements, where the estimation problem is reformulated as two loosely coupled linear subproblems. This paper is organized as follows. Section 2 derives the system dynamic model under the random sampling time and gives the estimation method based on Kalman filter. The convergence of the algorithm is proved in Section 3. The simulations and experiments are provided in Section 4. Finally, some concluding remarks are given in Section 5.

\section{System Model and Closed-Loop Estimation Method}

We begin with the continuous dynamic model of the moving target. Let $x, \dot{x}$, and $\ddot{x}$ be the target location, velocity, and acceleration along a generic direction, and the state is expressed as $x=[x, \dot{x}, \ddot{x}]^{T}$. Assume the nonzero mean acceleration satisfies $\ddot{x}(t)=\bar{a}(t)+a(t)$, where $\bar{a}(t)$ is the mean of acceleration in the interval $\left[\begin{array}{ll}0 & t\end{array}\right]$ and $a(t)$ is a zero mean first-order stationary Markov process with variance $\delta_{a}^{2}$. We have $\dot{a}(t)=-\alpha a(t)+w(t) ; \alpha$ is maneuver frequency and $w(t)$ is zero mean processing white noise with variance $\delta_{w}^{2}=2 \alpha \delta_{a}^{2}$. The parameter $\alpha=1 / \tau$ is the reciprocal of the maneuver time constant $\tau$ and thus depends on how long the maneuver lasts. For example, for an aircraft $\tau \approx 60 \mathrm{~s}$ for a lazy turn and $\tau \approx 10-20 \mathrm{~s}$ for an evasive maneuver. The parameter $\delta_{a}^{2}=E\left[a^{2}(t)\right]$ is the "instantaneous variance" of the acceleration.

Then, we can obtain the acceleration satisfying $\dot{\ddot{x}}(t)=$ $-\alpha \ddot{x}(t)+\alpha \bar{a}(t)+w(t)$ and the following state-space representation of the continuous time model can be obtained:

$$
\dot{x}(t)=A x(t)+U \bar{a}(t)+B w(t),
$$

where

$$
A=\left[\begin{array}{ccc}
0 & 1 & 0 \\
0 & 0 & 1 \\
0 & 0 & -\alpha
\end{array}\right], \quad U=\left[\begin{array}{l}
0 \\
0 \\
\alpha
\end{array}\right], \quad B=\left[\begin{array}{l}
0 \\
0 \\
1
\end{array}\right],
$$

and $w(t)$ is process noise with the covariance matrix given by $w(t) \sim N\left(0,2 \alpha \delta_{a}^{2}\right)$. Assume the measurement data is obtained at the sampling time $t_{i}$ and the measurement equation is as follows:

$$
z\left(t_{i}\right)=H\left(t_{i}\right) x\left(t_{i}\right)+v\left(t_{i}\right), \quad i=0,1,2, \ldots,
$$

where $H\left(t_{i}\right)$ is measurement matrix and $v\left(t_{i}\right)$ is measurement noise with the known variance $R$; that is, $v\left(t_{i}\right) \sim N(0, R)$.

2.1. Model Discretization. We can get the following by the differential equation (1):

$$
\begin{aligned}
x(t)= & e^{A\left(t-t_{0}\right)} x\left(t_{0}\right)+\int_{t_{0}}^{t} e^{A(t-\lambda)} U \bar{a}(\lambda) d \lambda \\
& +\int_{t_{0}}^{t} e^{A(t-\lambda)} B w(\lambda) d \lambda .
\end{aligned}
$$

We can see that for any known integration interval $\left[t_{0}, t\right]$, $x(t)$ can be gotten at any time $t$ if the initial state $x\left(t_{0}\right)$, the parameters $A, U, B, \bar{a}(t)$, and $w(t)$ in $\left[t_{0}, t\right]$ are known.

Consider the time interval from $t_{i-1}$ to $t_{i}$ and assume

$$
\begin{aligned}
\bar{a}(\lambda)=\bar{a}\left(t_{i-1}\right), \quad w(\lambda) & =w\left(t_{i-1}\right), \\
\lambda & \in\left[\begin{array}{ll}
t_{i-1} & t_{i}
\end{array}\right] ;
\end{aligned}
$$

we can have

$$
\begin{gathered}
\int_{t_{i-1}}^{t_{i}} e^{A\left(t_{i}-\lambda\right)} U \bar{a}(\lambda) d \lambda=\int_{t_{i-1}}^{t_{i}} e^{A\left(t_{i}-\lambda\right)} U d \lambda \bar{a}\left(t_{i-1}\right) \\
\int_{t_{i-1}}^{t_{i}} e^{A\left(t_{i}-\lambda\right)} B w(\lambda) d \lambda=\int_{t_{i-1}}^{t_{i}} e^{A\left(t_{i}-\lambda\right)} B d \lambda w\left(t_{i-1}\right) .
\end{gathered}
$$

Set $\mathrm{th}_{i}=t_{i}-t_{i-1}$; we have the system matrix as $A_{d}\left(t_{i-1}\right)=e^{A \mathrm{th}_{i}}, U_{d}\left(t_{i-1}\right)=\int_{t_{i-1}}^{t_{i}} e^{A\left(t_{i}-\lambda\right)} U d \lambda$ and the noise $w_{d}\left(t_{i-1}\right)=\int_{t_{i-1}}^{t_{i}} e^{A\left(t_{i}-\lambda\right)} B d \lambda w\left(t_{i-1}\right)$ with the covariance $Q_{d}\left(t_{i-1}\right)=E\left[w_{d}\left(t_{i-1}\right) w_{d}^{T}\left(t_{i-1}\right)\right]$.

Because the process matrix $A$ in (2) is not a full-rank matrix, we cannot calculate the matrix exponential $e^{A t_{i}}$ by the Lagrange-Hermite interpolation. Here, we use Laplace transform and have

$$
(s I-A)^{-1}=\left[\begin{array}{ccc}
s & -1 & 0 \\
0 & s & -1 \\
0 & 0 & s+\alpha
\end{array}\right]^{-1}=\left[\begin{array}{ccc}
\frac{1}{s} & \frac{1}{s^{2}} & \frac{1}{s^{2}(s+\alpha)} \\
0 & \frac{1}{s} & \frac{1}{s(s+\alpha)} \\
0 & 0 & \frac{1}{s+\alpha}
\end{array}\right] .
$$


The matrix exponential $e^{A t h_{i}}$ can be gotten by the inverse Laplace transform as

$$
A_{d}\left(t_{i-1}\right)=\left[\begin{array}{ccc}
1 & \mathrm{th}_{i} & \frac{\alpha \mathrm{th}_{i}-1+e^{-\alpha \mathrm{th}_{i}}}{\alpha^{2}} \\
0 & 1 & \frac{1-e^{-\alpha \mathrm{th}_{i}}}{\alpha} \\
0 & 0 & e^{-\alpha \mathrm{th}_{i}}
\end{array}\right]
$$

and by the similar approach, we can get the system parameter

$$
U_{d}\left(t_{i-1}\right)=\left[\begin{array}{c}
\frac{1}{\alpha}\left(-\mathrm{th}_{i}+\frac{\alpha \cdot \mathrm{th}_{i}^{2}}{2}+\frac{1-e^{-\alpha \cdot \mathrm{th}_{i}}}{\alpha}\right) \\
\operatorname{th}_{i}-\frac{1-e^{-\alpha \cdot \mathrm{th}_{i}}}{\alpha} \\
1-e^{-\alpha \cdot \mathrm{th}_{i}}
\end{array}\right]
$$

and the variance of $w_{d}\left(t_{i-1}\right)$ as

$$
\begin{aligned}
Q_{d}\left(t_{i-1}\right) & =E\left[w_{d}\left(t_{i-1}\right) w_{d}^{T}\left(t_{i-1}\right)\right] \\
& =2 \alpha \delta_{\alpha}^{2}\left[\begin{array}{lll}
q_{11} & q_{12} & q_{13} \\
q_{12} & q_{22} & q_{23} \\
q_{13} & q_{23} & q_{33}
\end{array}\right]
\end{aligned}
$$

with the parameters described as

$$
\begin{gathered}
q_{11}=\frac{1}{2 \alpha^{5}}\left[1-e^{-2 \alpha \cdot \mathrm{th}_{i}}+2 \alpha \cdot \mathrm{th}_{i}\right. \\
\left.+\frac{2 \alpha^{3} \mathrm{th}_{i}^{3}}{3}-2 \alpha^{2} \mathrm{th}_{i}^{2}-4 \alpha \cdot \mathrm{th}_{i} e^{-\alpha \cdot \mathrm{th}_{i}}\right] \\
q_{12}=\frac{1}{2 \alpha^{4}}\left[e^{-2 \alpha \cdot \mathrm{th}_{i}}+1-2 e^{-\alpha \cdot \mathrm{th}_{i}}\right. \\
\left.+2 \alpha \cdot \mathrm{th}_{i} e^{-\alpha \cdot \mathrm{th}_{i}}-2 \alpha \cdot \mathrm{th}_{i}+\alpha^{2} \mathrm{th}_{i}^{2}\right] \\
q_{13}=\frac{1}{2 \alpha^{3}}\left[1-e^{-2 \alpha \cdot \mathrm{th}_{i}}-2 \alpha \cdot \mathrm{th}_{i} e^{-\alpha \cdot \mathrm{th}_{i}}\right] \\
q_{22}=\frac{1}{2 \alpha^{3}}\left[4 e^{-\alpha \cdot \mathrm{th}_{i}}-3-e^{-2 \alpha \cdot \mathrm{th}_{i}}+2 \alpha \cdot \mathrm{th}_{i}\right] \\
q_{23}=\frac{1}{2 \alpha^{2}}\left[e^{-2 \alpha \cdot \mathrm{th}_{i}}+1-2 \alpha \cdot \mathrm{th}_{i}\right] \\
q_{33}=\frac{1}{2 \alpha}\left[1-e^{-2 \alpha \cdot \mathrm{th}_{i}}\right] .
\end{gathered}
$$

Then, we get the discrete state-space model of the tracking system as

$$
\begin{gathered}
x\left(t_{i}\right)=A_{d}\left(t_{i-1}\right) x\left(t_{i-1}\right)+U_{d}\left(t_{i-1}\right) \bar{a}\left(t_{i-1}\right)+w_{d}\left(t_{i-1}\right) \\
z\left(t_{i}\right)=H\left(t_{i}\right) x\left(t_{i}\right)+v\left(t_{i}\right),
\end{gathered}
$$

where $x=[x, \dot{x}, \ddot{x}]^{T}$ is the state of the system to be estimated and whose initial mean and covariance are known as $x_{0}$ and $P_{0}, w_{d}\left(t_{i}\right)$ and $v\left(t_{i}\right)$ are white noise with zero mean and independent of the initial state $x_{0}, z\left(t_{i}\right)$ is the measurement vector, $H\left(t_{i}\right)$ is measurement matrices, and $v\left(t_{i}\right)$ is measurement noise with known variance $R$. Until now, the irregular sampling is turned to the varying-parameter system. We can see the same sampling interval is just a particular case of the random sampling problem. Therefore, the model of the randomly sampling tracking is a general one.

2.2. System Parameters Estimation. Here, we assume the maneuver frequency $\alpha$ and the variance of the acceleration $\delta_{a}^{2}$ are not constant but variable and expressed as $\alpha_{i}$ and $\delta_{a i}^{2}$. From the processing model of (12), we have the discrete time equation of the acceleration as

$$
\ddot{x}\left(t_{i}\right)=\beta_{i} \ddot{x}\left(t_{i-1}\right)+\left(1-\beta_{i}\right) \bar{a}\left(t_{i-1}\right)+w^{a}\left(t_{i-1}\right),
$$

where $\beta_{i}=e^{-\alpha_{i} \mathrm{t}_{i}}$ and $w^{a}\left(t_{i-1}\right)$ is a zero mean white noise sequence with the variance

$$
\delta_{a w i}^{2}=\delta_{a i}^{2}\left(1-\beta_{i}^{2}\right) .
$$

$\alpha_{i}$ is the maneuver frequency at the sampling time $t_{i} \cdot \bar{a}\left(t_{i-1}\right)$ is the mean of one interval, so we have $\bar{a}\left(t_{i}\right)=\bar{a}\left(t_{i-1}\right)$. Set $a\left(t_{i}\right)=\ddot{x}\left(t_{i}\right)-\bar{a}\left(t_{i}\right)$; then we can obtain

$$
a\left(t_{i}\right)=\beta_{i} a\left(t_{i-1}\right)+w^{a}\left(t_{i-1}\right) .
$$

Consider the estimation of acceleration $\widehat{a}\left(t_{i}\right)$ is a random process; we have

$$
\bar{a}\left(t_{i-1}\right)=\frac{1}{i} \sum_{i=0}^{i-1} \widehat{\ddot{x}}\left(t_{i}\right),
$$

where $i$ is the number of data. For a first-order stationary Markov process (15), we have the statistics relation between the autocorrelation functions $r(0), r(1)$ with the parameters $\beta_{i}$ and $\delta_{a w i}^{2}$ by the Yule-Walker method [18]

$$
\begin{gathered}
r_{i}(0)=\frac{1}{i} \sum_{i=0}^{i-1} \widehat{a}\left(t_{i}\right) \widehat{a}\left(t_{i}\right) \\
r_{i}(1)=\frac{1}{i} \sum_{i=1}^{i-1} \widehat{a}\left(t_{i}\right) \widehat{a}\left(t_{i-1}\right) \\
\beta_{i}=\frac{r_{i}(1)}{r_{i}(0)} \\
\delta_{a w i}^{2}=r_{i}(0)-\beta_{i} r_{i}(1) .
\end{gathered}
$$

Next, we can get $\alpha_{i}$ and $\delta_{a i}^{2}$ by $\delta_{a i}^{2}=\delta_{a w i}^{2} /\left(1-\beta_{i}^{2}\right), \alpha_{i}=$ $\ln \beta_{i} /-\mathrm{th}_{i}$, and then get the system parameters $A_{d}\left(t_{i-1}\right)$, $U_{d}\left(t_{i-1}\right)$, and $Q_{d}\left(t_{i-1}\right)$ in process function (12).

2.3. Algorithm Summary. Now we summarize the closedloop estimation algorithm for the randomly sampled measurements as follows. 
(1) Initialization $(i=0)$. Consider

$$
\begin{gathered}
\hat{x}\left(t_{0} \mid t_{0}\right)=x_{0}, \\
P\left(t_{0} \mid t_{0}\right)=P_{0}, \alpha_{0}, \delta_{a 0}^{2}, \bar{a}\left(t_{0}\right), \\
r_{0}\left(t_{0}\right)=\ddot{x}_{0} \cdot \ddot{x}_{0}, \quad r_{0}\left(t_{1}\right)=\ddot{x}_{0} .
\end{gathered}
$$

(2) Recursion $(i:=i+1)$

(a) System update: set $\mathrm{th}_{i}=t_{i}-t_{i-1}$ and the system parameter as

$$
\begin{gathered}
\widehat{A}_{d}\left(t_{i-1}\right)=\left[\begin{array}{ccc}
1 & \mathrm{th}_{i} & \frac{\alpha_{i} \mathrm{th}_{i}-1+e^{-\alpha_{i} \mathrm{th}_{i}}}{\alpha_{i}^{2}} \\
0 & 1 & \frac{1-e^{-\alpha_{i} \mathrm{th}_{i}}}{\alpha_{i}} \\
0 & 0 & e^{-\alpha_{i} \mathrm{th}_{i}}
\end{array}\right] \\
\widehat{U}_{d}\left(t_{i-1}\right)=\left[\begin{array}{c}
\frac{1}{\alpha_{i}}\left(-\mathrm{th}_{i}+\frac{\alpha_{i} \cdot \mathrm{th}_{i}^{2}}{2}+\frac{1-e^{-\alpha_{i} \cdot \mathrm{th}_{i}}}{\alpha_{i}}\right) \\
\mathrm{th}_{i}-\frac{1-e^{-\alpha_{i} \cdot \mathrm{th}_{i}}}{\alpha_{i}} \\
1-e^{-\alpha_{i} \cdot \mathrm{th}_{i}}
\end{array}\right]
\end{gathered}
$$

and the variance of the $w_{d}\left(t_{i-1}\right)$ as

$$
\begin{aligned}
\widehat{Q}_{d}\left(t_{i-1}\right) & =E\left[w_{d}\left(t_{i-1}\right) w_{d}^{T}\left(t_{i-1}\right)\right] \\
& =2 \alpha_{i} \delta_{\alpha i}^{2}\left[\begin{array}{lll}
q_{11} & q_{12} & q_{13} \\
q_{12} & q_{22} & q_{23} \\
q_{13} & q_{23} & q_{33}
\end{array}\right]
\end{aligned}
$$

with parameters described as

$$
\begin{aligned}
& q_{11}=\frac{1}{2 \alpha_{i}^{5}}\left[1-e^{-2 \alpha_{i} \cdot \mathrm{th}_{i}}+2 \alpha_{i} \cdot \mathrm{th}_{i}\right. \\
& \left.+\frac{2 \alpha_{i}^{3} \operatorname{th}_{i}^{3}}{3}-2 \alpha_{i}^{2} \operatorname{th}_{i}^{2}-4 \alpha_{i} \cdot \operatorname{th}_{i} e^{-\alpha \cdot \mathrm{th}_{i}}\right] \\
& q_{12}=\frac{1}{2 \alpha_{i}^{4}}\left[e^{-2 \alpha_{i} \cdot \mathrm{th}_{i}}+1-2 e^{-\alpha_{i} \cdot \mathrm{th}_{i}}\right. \\
& \left.+2 \alpha_{i} \cdot \operatorname{th}_{i} e^{-\alpha_{i} \cdot \mathrm{th}_{i}}-2 \alpha_{i} \cdot \mathrm{th}_{i}+\alpha_{i}^{2} \operatorname{th}_{i}^{2}\right] \\
& q_{13}=\frac{1}{2 \alpha_{i}^{3}}\left[1-e^{-2 \alpha_{i} \cdot \mathrm{th}_{i}}-2 \alpha_{i} \cdot \mathrm{th}_{i} e^{-\alpha_{i} \cdot \mathrm{th}_{i}}\right] \\
& q_{22}=\frac{1}{2 \alpha_{i}^{3}}\left[4 e^{-\alpha_{i} \cdot \mathrm{th}_{i}}-3-e^{-2 \alpha_{i} \cdot \mathrm{th}_{i}}+2 \alpha_{i} \cdot \mathrm{th}_{i}\right] \\
& q_{23}=\frac{1}{2 \alpha_{i}^{2}}\left[e^{-2 \alpha_{i} \cdot \mathrm{th}_{i}}+1-2 \alpha_{i} \cdot \mathrm{th}_{i}\right] \\
& q_{33}=\frac{1}{2 \alpha_{i}}\left[1-e^{-2 \alpha_{i} \cdot \mathrm{th}_{i}}\right] .
\end{aligned}
$$

(b) State prediction: consider

$$
\begin{aligned}
& \widehat{x}\left(t_{i} \mid t_{i-1}\right) \\
& \quad=\widehat{A}_{d}\left(t_{i-1}\right) \hat{x}\left(t_{i-1} \mid t_{i-1}\right)+\widehat{U}_{d}\left(t_{i-1}\right) \bar{a}\left(t_{i-1}\right) \\
& P\left(t_{i} \mid t_{i-1}\right) \\
& \quad=\widehat{A}_{d}\left(t_{i-1}\right) P\left(t_{i-1} \mid t_{i-1}\right) \widehat{A}_{d}^{T}\left(t_{i-1}\right)+\widehat{Q}_{d}\left(t_{i-1}\right) .
\end{aligned}
$$

(c) State update: consider

$$
\begin{aligned}
& \widehat{x}\left(t_{i} \mid t_{i}\right) \\
& =\hat{x}\left(t_{i} \mid t_{i-1}\right)+K\left(t_{i}\right)\left[z\left(t_{i}\right)-H\left(t_{i}\right) \hat{x}\left(t_{i} \mid t_{i-1}\right)\right] \\
& K\left(t_{i}\right) \\
& =P\left(t_{i} \mid t_{i-1}\right) H^{T}\left(t_{i}\right) \\
& \quad \times\left[H\left(t_{i}\right) P\left(t_{i} \mid t_{i-1}\right) H^{T}\left(t_{i}\right)+R\left(t_{i}\right)\right]^{-1} \\
& \quad P\left(t_{i} \mid t_{i}\right)=\left[I-K\left(t_{i}\right) H\left(t_{i}\right)\right] P\left(t_{i} \mid t_{i-1}\right) .
\end{aligned}
$$

(d) Parameter adaptation: the mean of the acceleration

$$
\bar{a}\left(t_{i-1}\right)=\frac{1}{i} \sum_{i=0}^{i-1} \widehat{\ddot{x}}\left(t_{i} \mid t_{i}\right) .
$$

When $i \leq K_{0}$, the maneuver frequency $\alpha_{i}$ is set to $\alpha_{0}$ and the covariance of the noise $\delta_{a i}^{2}$ is gotten by the following:

$$
\delta_{\alpha i}^{2}= \begin{cases}\frac{4-\pi}{\pi}\left[a_{M}-\widehat{\ddot{x}}\left(t_{i} \mid t_{i}\right)\right]^{2} & \text { when } \widehat{\ddot{x}}\left(t_{i} \mid t_{i}\right)>0 \\ \frac{4-\pi}{\pi}\left[\widehat{\ddot{x}}\left(t_{i} \mid t_{i}\right)-a_{-M}\right]^{2} & \text { when } \widehat{\ddot{x}}\left(t_{i} \mid t_{i}\right)<0 \\ \text { a small positive constant } & \text { when } \widehat{\ddot{x}}\left(t_{i} \mid t_{i}\right)=0 .\end{cases}
$$

When $i>K_{0}$, the parameter is updated by the following

$$
\begin{gathered}
\widehat{a}\left(t_{i}\right)=\widehat{\ddot{x}}\left(t_{i} \mid t_{i}\right)-\bar{a}\left(t_{i}\right) \\
r_{i}(1)=r_{i-1}(1)+\frac{1}{i}\left[\widehat{a}\left(t_{i}\right) \widehat{a}\left(t_{i-1}\right)-r_{i-1}(1)\right] \\
r_{i}(0)=r_{i-1}(0)+\frac{1}{i}\left[\widehat{a}\left(t_{i}\right) \widehat{a}\left(t_{i}\right)-r_{i-1}(0)\right] \\
\beta_{i}=\frac{r_{i}(1)}{r_{i}(0)} \quad \delta_{a w i}^{2}=r_{i}(0)-\beta_{i} r_{i}(1) \\
\delta_{a i}^{2}=\frac{\delta_{a w i}^{2}}{1-\beta_{i}^{2}} \quad \alpha_{i}=\frac{\ln \beta_{i}}{-\mathrm{th}_{i}} .
\end{gathered}
$$

The irregular sampling time $t_{i-1}, t_{i}$ and the interval th reflect in the time-varying parameters of the system, so we can conclude that the Kalman filter shown in (23)-(33) based on system (12) with system parameters (19)-(22) can obtain the same estimation performance as regular sampling Kalman filter. 


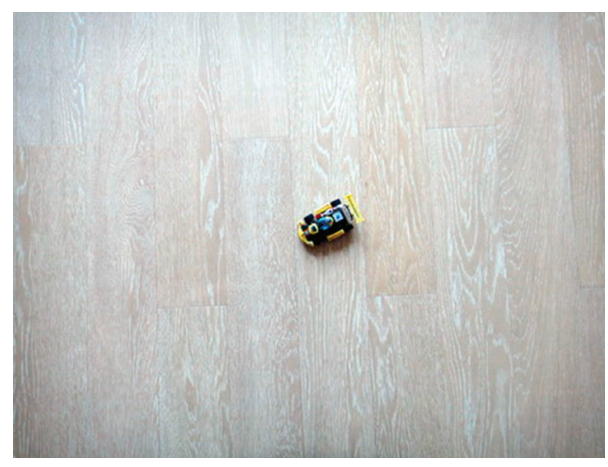

FIGURE 1: The video with simple background and one target.

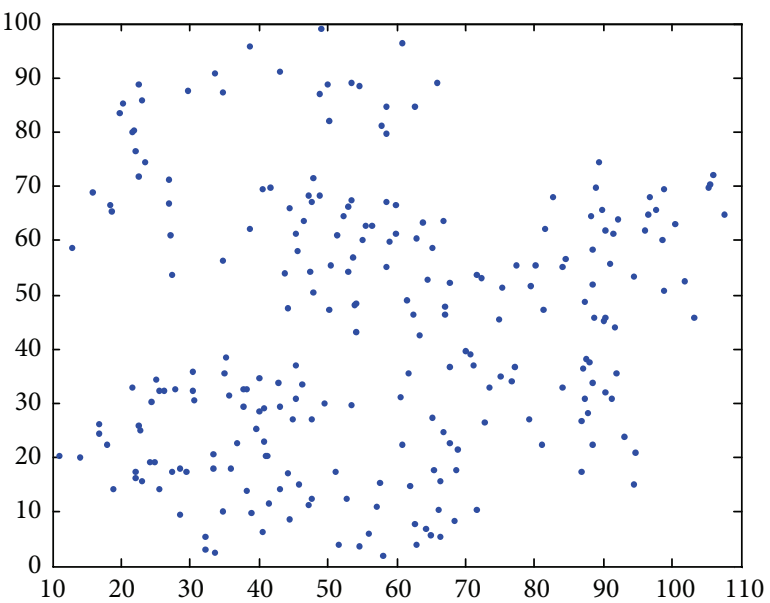

FIGURE 2: The measurement of maneuvering target got from the video.

\section{Proof of the Convergence}

Based on the closed-loop estimation algorithm (18)-(33), we can see that the parameter used to estimate state is an estimated one and similarly the estimated states to calculate parameters $\alpha_{i}$ and $\delta_{a i}^{2}$ have estimation errors too. Therefore, it is important to guarantee the convergence of the estimation of the states and parameters.

From (27), (29), and (33), we know if the estimation $\widehat{\ddot{x}}\left(t_{i} \mid\right.$ $t_{i}$ ) increased suddenly, $\widehat{a}\left(t_{i}\right)$ will increase greatly because the mean changes less than $\widehat{\ddot{x}}\left(t_{i} \mid t_{i}\right)$, and $\delta_{\text {awi }}^{2}$ becomes large too. Then, a very large positive $\delta_{a i}^{2}$ will be obtained, and $\widehat{Q}_{d}\left(t_{i}\right)$ will also contain a large number of elements (here, we call it a big matrix). From the Riccati equation of Kalman filter

$$
\begin{aligned}
P\left(t_{i+1} \mid t_{i}\right) & \\
=\widehat{A}_{d}\left(t_{i}\right)\{ & P\left(t_{i} \mid t_{i-1}\right)-P\left(t_{i} \mid t_{i-1}\right) H^{T}\left(t_{i}\right) \\
& \times\left[H\left(t_{i}\right) P\left(t_{i} \mid t_{i-1}\right) H^{T}\left(t_{i}\right)+R\left(t_{i}\right)\right]^{-1} \\
& \left.\times H\left(t_{i}\right) P\left(t_{i} \mid t_{i-1}\right)\right\} \widehat{A}_{d}^{T}\left(t_{i}\right)+\widehat{Q}_{d}\left(t_{i}\right),
\end{aligned}
$$

we find that $P\left(t_{i+1} \mid t_{i}\right)$ will be a big matrix if $\widehat{Q}_{d}\left(t_{i}\right)$ is a big one, and $K\left(t_{i+1}\right)$ will increase greatly. As a result, the esti-

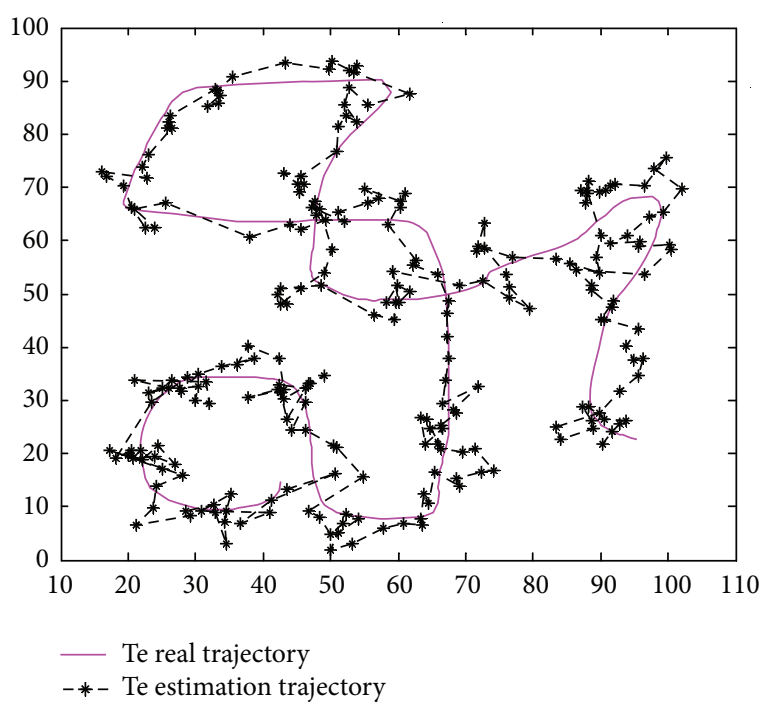

FIGURE 3: The real trajectory and the estimation trajectory.

mation state $\widehat{x}\left(t_{i} \mid t_{i}\right)=\widehat{x}\left(t_{i} \mid t_{i-1}\right)+K\left(t_{i}\right)\left[z\left(t_{i}\right)-H\left(t_{i}\right) \widehat{x}\left(t_{i} \mid\right.\right.$ $\left.t_{i-1}\right)$ ] will be a big matrix too. This trend results in positive feedback loops, which means $\widehat{x}\left(t_{i} \mid t_{i}\right)$ will become larger and larger, and finally, divergence. We give the following theorem to guarantee the algorithm convergence.

Theorem 1. The estimation $\widehat{x}\left(t_{i+1} \mid t_{i+1}\right)$ is bounded if the variance of the target acceleration $\delta_{a i}^{2}$ has an upper bound; that is, there is a positive $\delta_{0}^{2}$ satisfying $\delta_{a i}^{2} \leq \delta_{0}^{2}$.

Proof. We firstly consider maneuvering frequency $\alpha_{i}$. From (19), (21), and (22), we know if $\alpha_{i} \rightarrow 0$ and $\delta_{a i}^{2} \leq \delta_{0}^{2}$, the target has the constant acceleration maneuvering, and the system model is the constant acceleration model with the parameter as follows

$$
\begin{gathered}
\widehat{A}_{d}\left(t_{i-1}\right) \longrightarrow \bar{A}_{d}\left(t_{i-1}\right)=\left[\begin{array}{ccc}
1 & \mathrm{th}_{i} & \frac{\mathrm{th}_{i}^{2}}{2} \\
0 & 1 & \mathrm{th}_{i} \\
0 & 0 & 1
\end{array}\right] \\
\widehat{Q}_{d}\left(t_{i-1}\right) \longrightarrow \bar{Q}_{d}\left(t_{i-1}\right)=\delta_{\alpha i}^{2}\left[\begin{array}{ccc}
\frac{\mathrm{th}_{i}^{5}}{20} & \frac{\mathrm{th}_{i}^{4}}{8} & \frac{\mathrm{th}_{i}^{3}}{6} \\
\frac{\mathrm{th}_{i}^{4}}{8} & \frac{\mathrm{th}_{i}^{3}}{3} & \frac{\mathrm{th}_{i}^{2}}{2} \\
\frac{\mathrm{th}_{i}^{3}}{6} & \frac{\mathrm{th}_{i}^{2}}{2} & \mathrm{th}_{i}
\end{array}\right] .
\end{gathered}
$$

If $\alpha_{i} \rightarrow \infty$ and $\delta_{a i}^{2} \leq \delta_{0}^{2}$, we can get the system parameter matrix such as $\widehat{A}_{d}\left(t_{i-1}\right) \rightarrow \overline{\bar{A}}_{d}\left(t_{i-1}\right)=\left[\begin{array}{ccc}1 & \text { th }_{i} & 0 \\ 0 & 1 & 0 \\ 0 & 0 & 1\end{array}\right]$ and $\widehat{Q}_{d}\left(t_{i-1}\right) \rightarrow \overline{\bar{Q}}_{d}\left(t_{i-1}\right)=\delta_{\alpha i}^{2}\left[\begin{array}{lll}0 & 0 & 0 \\ 0 & 0 & 0 \\ 0 & 0 & 1\end{array}\right]$. Therefore, we can see that $\widehat{A}_{d}\left(t_{i-1}\right)$ and $\widehat{Q}_{d}\left(t_{i-1}\right)$ are the monotonic matrix with finite value elements. 


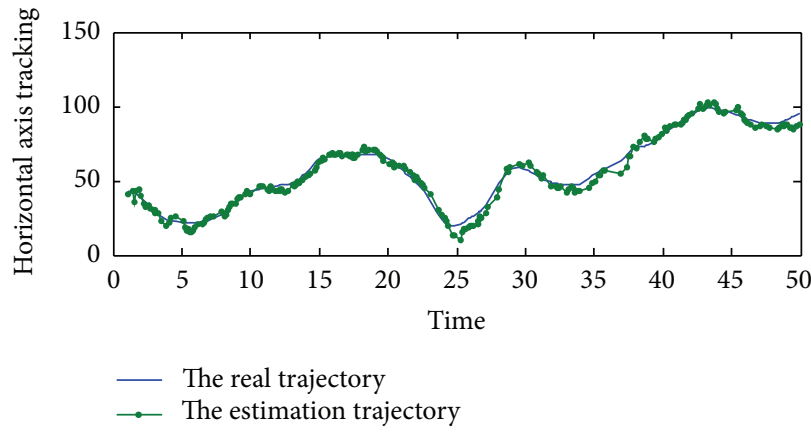

(a)

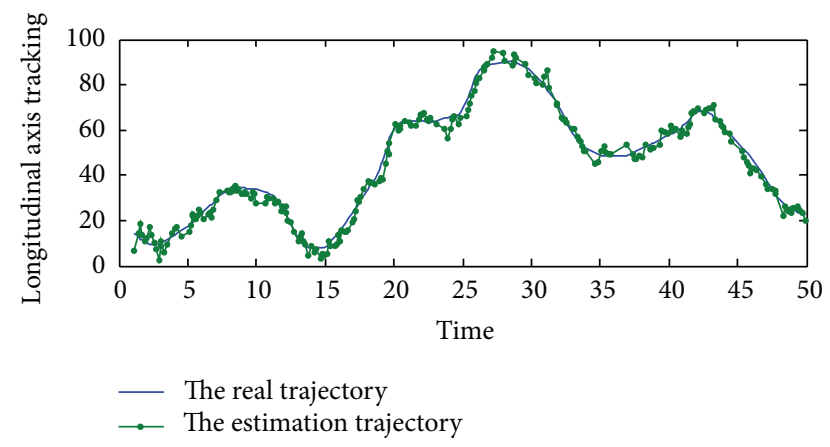

(b)

FIgURE 4: The estimations of horizontal and longitudinal axis.

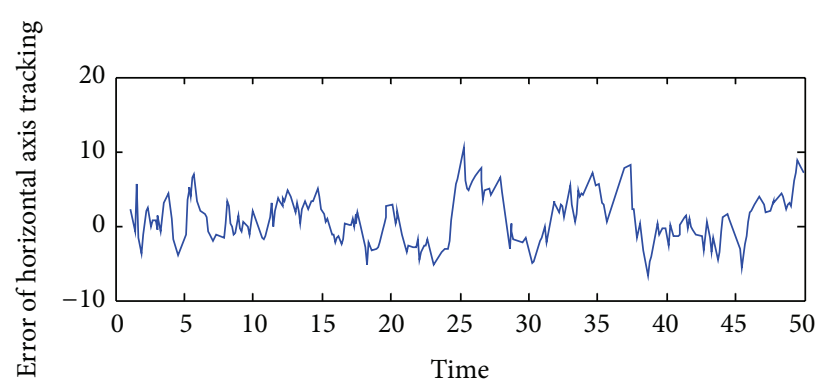

(a)

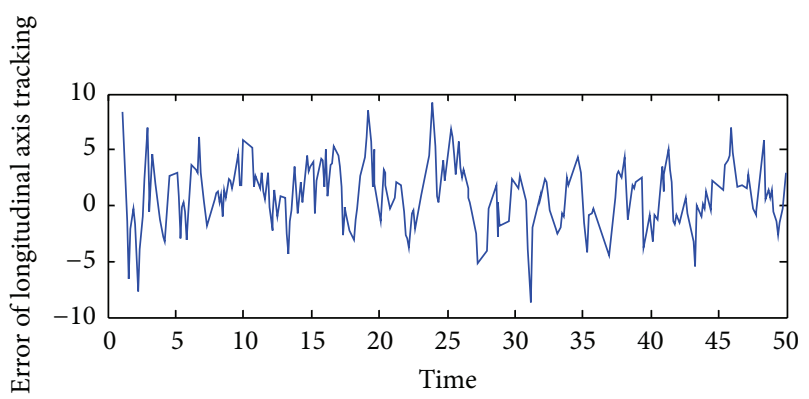

(b)

FIGURE 5: The location estimation errors.

Then, we consider the solution of Riccati equation (34) on the condition that the system parameter matrix has errors, such as $\widehat{A}_{d}=\widetilde{A}_{d}+\Delta A_{d}$ and $\widehat{Q}_{d}=\widetilde{Q}_{d}+\Delta Q_{d}$, where $\widetilde{A}_{d}$ and $\widetilde{Q}_{d}$ are the actual system parameters and $\Delta A_{d}$ and $\Delta Q_{d}$ are the errors of the system parameter. Unlike the research about the uncertainty system, here we do not know the actual system matrices $\widetilde{A}_{d}$ and $\widetilde{Q}_{d}$, but we can know the upper bound of the system parameters $\widehat{A}_{d}\left(t_{i-1}\right)$ and $\widehat{Q}_{d}\left(t_{i-1}\right)$, when $\delta_{a i}^{2} \leq \delta_{0}^{2}$, such as
The perturbed discrete algebraic Riccati equation is as follows:

$$
\begin{aligned}
P= & \widehat{A}_{d}\left(t_{i}\right) P \widehat{A}_{d}^{T}\left(t_{i}\right) \\
& -\widehat{A}_{d}\left(t_{i}\right) P H^{T}\left(t_{i}\right)\left[H\left(t_{i}\right) P H^{T}\left(t_{i}\right)+R\left(t_{i}\right)\right]^{-1} \\
& \times H\left(t_{i}\right) P \widehat{A}_{d}^{T}\left(t_{i}\right)+\widehat{Q}_{d}\left(t_{i}\right) .
\end{aligned}
$$

We know that (37) is equal to

$$
\begin{aligned}
P= & \widehat{A}_{d}\left(t_{i}\right)\left(P^{-1}+H^{T}\left(t_{i}\right) R\left(t_{i}\right) H\left(t_{i}\right)\right)^{-1} \\
& \times \widehat{A}_{d}^{T}\left(t_{i}\right)+\widehat{Q}_{d}\left(t_{i}\right) .
\end{aligned}
$$

Then, for any vector $s$, we have

$$
\begin{aligned}
& s^{T} P s \\
& \qquad s^{T}\left[\widehat{A}_{d}\left(t_{i}\right)\left(P^{-1}+H^{T}\left(t_{i}\right) R\left(t_{i}\right) H\left(t_{i}\right)\right)^{-1}\right. \\
& \left.\quad \times \widehat{A}_{d}^{T}\left(t_{i}\right)+\widehat{Q}_{d}\left(t_{i}\right)\right] s \\
& \leq \lambda_{1}\left(P^{-1}+H^{T}\left(t_{i}\right) R\left(t_{i}\right) H\left(t_{i}\right)\right)^{-1} s^{T} \widehat{A}_{d}\left(t_{i}\right) \widehat{A}_{d}^{T}\left(t_{i}\right) s \\
& \quad+s^{T} \widehat{Q}_{d}\left(t_{i}\right) s,
\end{aligned}
$$




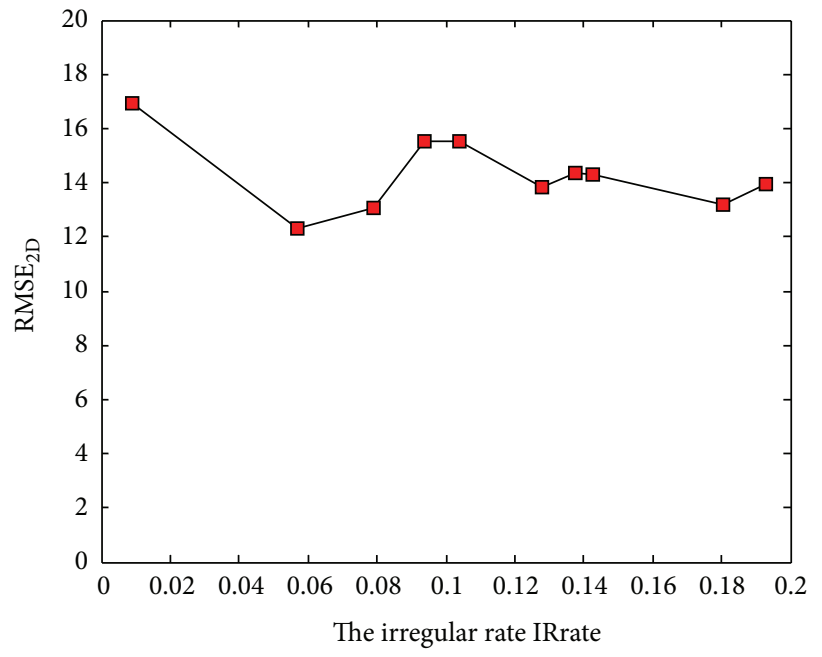

FIGURE 6: The relation between $\mathrm{RMSE}_{2 \mathrm{D}}$ and IRrate.

where $\lambda_{1}(X)$ is the maximum eigenvalue. By the relation of vector eigenvalue $\lambda_{m}\left(X^{-1}\right)=\lambda_{M-m+1}^{-1}(X)$, where $\lambda_{1}(X) \geq$ $\lambda_{2}(X) \geq \cdots \geq \lambda_{M}(X)$, we have

$$
s^{T} P s \leq \frac{s^{T} \widehat{A}_{d}\left(t_{i}\right) \widehat{A}_{d}^{T}\left(t_{i}\right) s}{\lambda_{M}\left(P^{-1}+H^{T}\left(t_{i}\right) R\left(t_{i}\right) H\left(t_{i}\right)\right)}+s^{T} \widehat{Q}_{d}\left(t_{i}\right) s .
$$

That is,

$$
P \leq \frac{\widehat{A}_{d}\left(t_{i}\right) \widehat{A}_{d}^{T}\left(t_{i}\right)}{\lambda_{M}\left(P^{-1}+H^{T}\left(t_{i}\right) R\left(t_{i}\right) H\left(t_{i}\right)\right)}+\widehat{Q}_{d}\left(t_{i}\right) .
$$

We have

$$
\begin{aligned}
\lambda_{M} & \left(P^{-1}+H^{T}\left(t_{i}\right) R\left(t_{i}\right) H\left(t_{i}\right)\right) \\
& \geq \frac{1}{\lambda_{1}(P)}+\lambda_{M}\left(H^{T}\left(t_{i}\right) R\left(t_{i}\right) H\left(t_{i}\right)\right) .
\end{aligned}
$$

Then, by (41) and (42), we have

$$
\begin{aligned}
P & \leq \frac{\widehat{A}_{d}\left(t_{i}\right) \widehat{A}_{d}^{T}\left(t_{i}\right)}{\left(1 / \lambda_{1}(P)\right)+\lambda_{M}\left(H^{T}\left(t_{i}\right) R\left(t_{i}\right) H\left(t_{i}\right)\right)}+\widehat{Q}_{d}\left(t_{i}\right) \\
& =\frac{\lambda_{1}(P) \widehat{A}_{d}\left(t_{i}\right) \widehat{A}_{d}^{T}\left(t_{i}\right)}{1+\lambda_{1}(P) \lambda_{M}\left(H^{T}\left(t_{i}\right) R\left(t_{i}\right) H\left(t_{i}\right)\right)}+\widehat{Q}_{d}\left(t_{i}\right) \\
& \leq \frac{\lambda_{1}(P) A_{\text {upper }}\left(t_{i}\right) A_{\text {upper }}^{T}\left(t_{i}\right)}{1+\lambda_{1}(P) \lambda_{M}\left(H^{T}\left(t_{i}\right) R\left(t_{i}\right) H\left(t_{i}\right)\right)}+Q_{\text {upper }}\left(t_{i}\right) .
\end{aligned}
$$

Next, by the relation of Hermite matrix and its eigenvalue, we have

$$
\begin{aligned}
\lambda_{1}(P) \leq & \frac{\lambda_{1}(P) \lambda_{1}\left(A_{\text {upper }}\left(t_{i}\right) A_{\text {upper }}^{T}\left(t_{i}\right)\right)}{1+\lambda_{1}(P) \lambda_{M}\left(H^{T}\left(t_{i}\right) R\left(t_{i}\right) H\left(t_{i}\right)\right)} \\
& +\lambda_{1}\left(Q_{\text {upper }}\left(t_{i}\right)\right) .
\end{aligned}
$$

Then, we have

$$
\begin{aligned}
& \lambda_{1}^{2}(P) \lambda_{M}\left(H^{T}\left(t_{i}\right) R\left(t_{i}\right) H\left(t_{i}\right)\right) \\
& +\lambda_{1}(P)\left[1-\lambda_{1}\left(A_{\text {upper }}\left(t_{i}\right) A_{\text {upper }}^{T}\left(t_{i}\right)\right)\right. \\
& \left.\quad-\lambda_{M}\left(H^{T}\left(t_{i}\right) R\left(t_{i}\right) H\left(t_{i}\right)\right) \lambda_{1}\left(Q_{\text {upper }}\left(t_{i}\right)\right)\right] \\
& \quad-\lambda_{1}\left(Q_{\text {upper }}\left(t_{i}\right)\right) \leq 0 .
\end{aligned}
$$

Assume that $\lambda_{M}\left(H^{T}\left(t_{i}\right) R\left(t_{i}\right) H\left(t_{i}\right)\right)>0$ and set

$$
\begin{aligned}
1 & -\lambda_{1}\left(A_{\text {upper }}\left(t_{i}\right) A_{\text {upper }}^{T}\left(t_{i}\right)\right) \\
& -\lambda_{M}\left(H^{T}\left(t_{i}\right) R\left(t_{i}\right) H\left(t_{i}\right)\right) \lambda_{1}\left(Q_{\text {upper }}\left(t_{i}\right)\right)=p_{1} .
\end{aligned}
$$

We have the following solution of (45):

$\lambda_{1}(P)$

$$
\leq \frac{-p_{1}+\sqrt{p_{1}^{2}+4 \lambda_{M}\left(H^{T}\left(t_{i}\right) R\left(t_{i}\right) H\left(t_{i}\right)\right) \lambda_{1}\left(Q_{\text {upper }}\left(t_{i}\right)\right)}}{2 \lambda_{M}\left(H^{T}\left(t_{i}\right) R\left(t_{i}\right) H\left(t_{i}\right)\right)} .
$$

Therefore, we can conclude that the maximum eigenvalue of estimation covariance $P$ has the upper bound shown as (47) if $\delta_{a i}^{2} \leq \delta_{0}^{2}$.

If one step predictive covariance is bounded, that is, $\left|P\left(t_{i} \mid t_{i-1}\right)\right| \leq P_{0}$, then we know $P\left(t_{i+1} \quad \mid t_{i}\right)$ must be bounded by (47) with the fact that $\left|\widehat{Q}_{d}\left(t_{i}\right)\right| \leq Q_{0}$. And based on (25), we know $K\left(t_{i+1}\right)$ must be a bounded matrix and $\widehat{x}\left(t_{i+1} \mid t_{i+1}\right)$ must be bounded too.

\section{Simulations and Experiments}

4.1. The Estimation by Different Extraction Rate and Irregular Rate. The method here is applied to a two-dimensional 

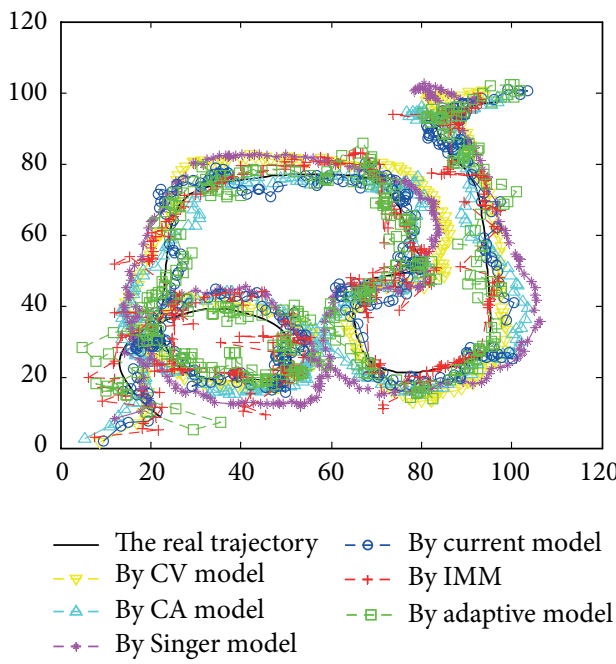

(a)

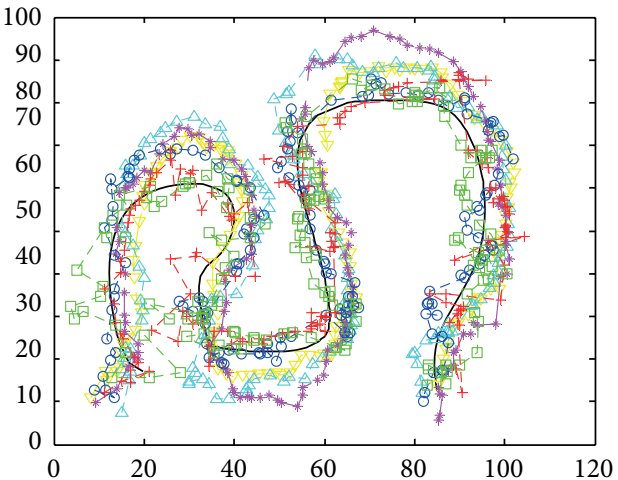

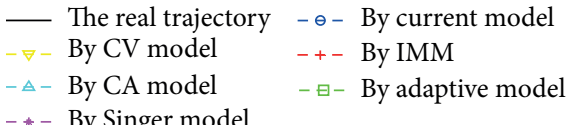

(c)

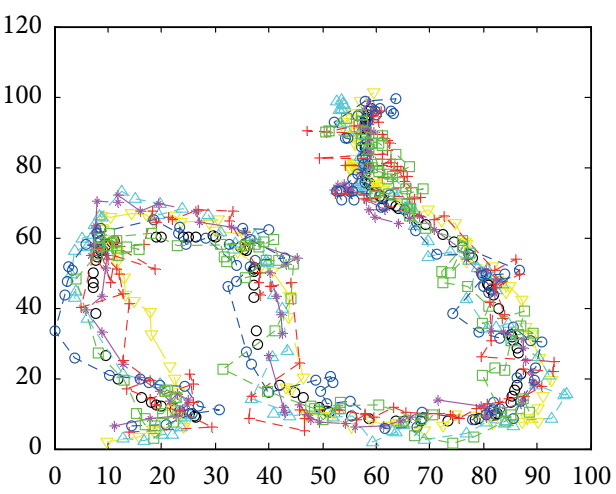

- The real trajectory $\quad-\theta-$ By current model By CV model

$-\triangle-$ By CA model

-* By Singer model

(e)

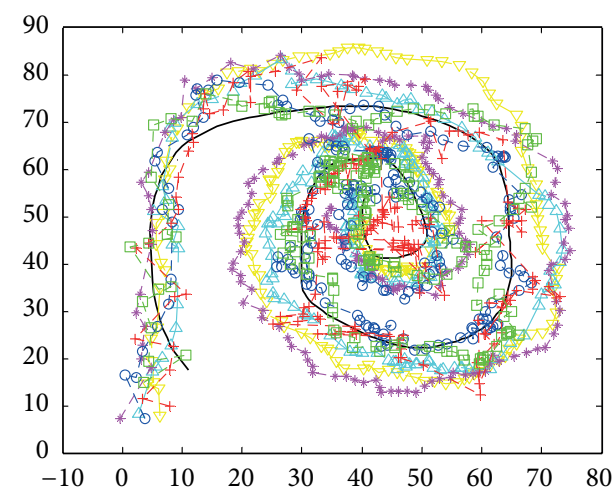

- The real trajectory - $-\theta-$ By current model By CV model $\quad-+-$ By IMM

$-\triangle-$ By CA model $\quad--_{-}$By adaptive model - * By Singer model

(b)

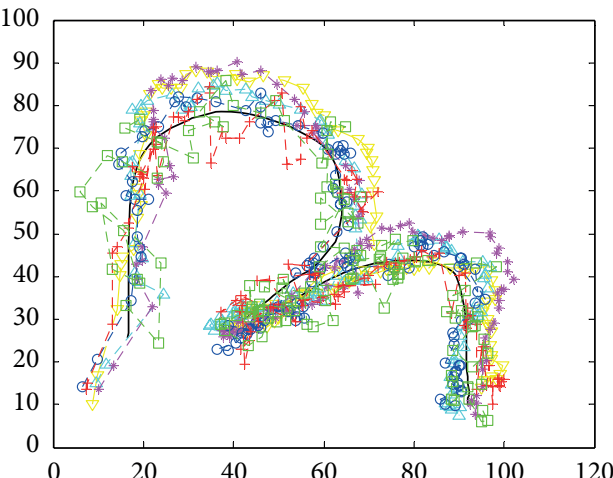

$$
\begin{array}{ll}
- \text { The real trajectory } & -\bullet-\text { By current model } \\
-\nabla-\text { By CV model } & -+- \text { By IMM } \\
-\Delta-\text { By CA model } & -\bullet-\text { By adaptive model } \\
-*-\text { By Singer model } &
\end{array}
$$

(d)

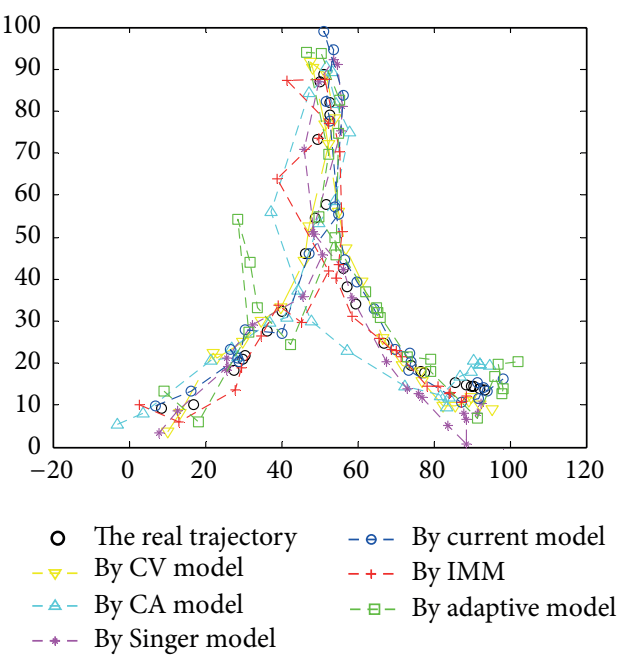

(f)

FIgURE 7: The tracking results for videos. 


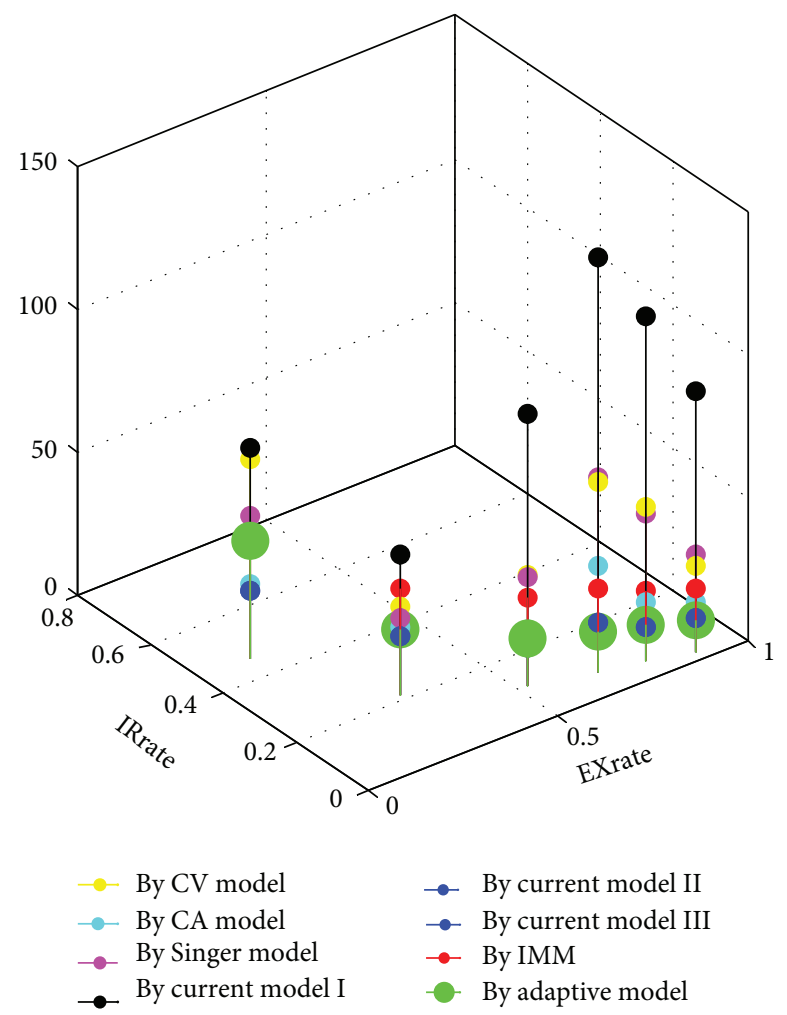

FIgURE 8: RMSE $_{2 \mathrm{D}}$ under different EXrate and IRrate.

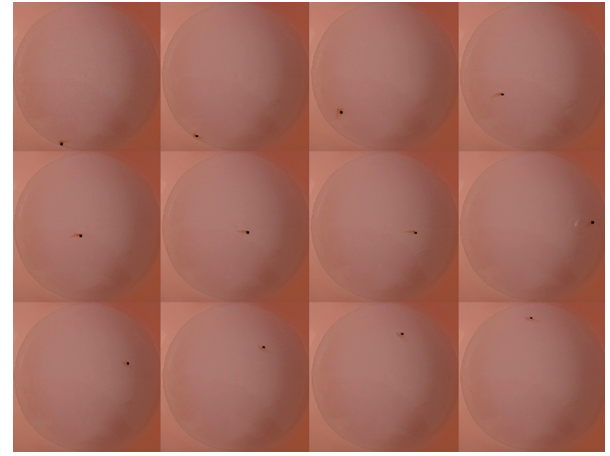

FIGURE 9: The tracking results in number 1, 27, 40, 65, 74, 97, 128, 129, $158,181,189$, and 226 frames.

planar video tracking. Here, as a tracking problem, we just use the simple background and one target. The video gotten by the Image Capture Test Bed is shown in Figure 1.

We control the car maneuvering on the test bed and catch the images of target movement by a stationary camera. For every image of the video, the target is extracted based on the color and then we get the measurement data of maneuvering target on the Image Capture Test Bed like Figure 2.

We know that the camera catches the image under the same interval, and that will produce large amounts of image data. If we can use some of images in the video for tracking, the image storage and computation cost will greatly reduce. But "using some of images" means that the measurements no longer have the same sampling interval. Here, define the Extraction Rate as

\section{EXrate}

$$
=\frac{\text { extracted number of images from the video }}{\text { total number of images in the video }} \times 100 \%
$$

to describe the image compression rate. And define the Irregular Rate to measure the sampling interval as

$$
\text { IRrate }=\frac{\sum_{i=1}^{N}\left|\mathrm{th}_{i}-\sum_{i=1}^{N} \mathrm{th}_{i}\right|}{N} .
$$

The state for the target in the $2 \mathrm{D}$ space is $x(k)=$ $\left[\begin{array}{llllll}x(k) & \dot{x}(k) & \ddot{x}(k) & y(k) & \dot{y}(k) & \ddot{y}(k)\end{array}\right]$. The initial state estimate $x_{0}$ and covariance $P_{0}$ are assumed to be $x_{0}=$ $\left[\begin{array}{llllll}x(0) & 0 & 0 & y(0) & 0 & 0\end{array}\right]^{T}$ and $P_{0}=\operatorname{diag}(10,10,10,10,10,10)$.

We extract 243 images from a video with 491 images where EXrate $=49.49 \%$ and IRrate $=0.1043$ and by the algorithm developed with the initial parameters $\alpha_{0}=1 / 20$, $\delta_{a 0}^{2}=10, \bar{a}_{0}=0, \alpha_{M}=3, K_{0}=3$, we get the estimation of trajectory with estimation covariance 10.0881 along the horizontal axis and 8.1660 along the vertical axis, shown in Figure 3. The estimation trajectories of horizontal and longitudinal axis is shown in Figure 4 and the estimation error are shown in Figure 5.

To illustrate how the irregular rate affects estimation performance, the algorithm is used to estimate the target 
TABLE 1: The different irregular rate for 10 cases.

\begin{tabular}{lccccccccc}
\hline Case 1 & Case 2 & Case 3 & Case 4 & Case 5 & Case 6 & Case 7 & Case 8 & Case 9 & Case 10 \\
\hline 0.01 & 0.06 & 0.08 & 0.09 & 0.10 & 0.13 & 0.14 & 0.14 & 0.18 & 0.19 \\
\hline
\end{tabular}

trajectory under different Irregular Rate (shown in Table 1) with the same Extraction Rate, EXrate $=49.86 \%$. The RMSE position is defined as RMSE ${ }_{2 \mathrm{D}}=\sqrt{\mathrm{RMSE}_{H}^{2}+\mathrm{RMSE}_{L}^{2}}$, where $\mathrm{RMSE}_{H}$ and $\mathrm{RMSE}_{L}$ are the root-mean square errors (RMSE) of position for horizontal and longitudinal axis, respectively. The relation between $\mathrm{RMSE}_{2 \mathrm{D}}$ and IRrate is shown in Figure 6. We can see that the Irregular Rate affects the estimation performance very little. The Irregular Rate changes 21 times almost from 0.0088 in Case 1 to 0.1928 in Case 10, but $\mathrm{RMSE}_{2 \mathrm{D}}$ is about 14 for all IRrate. We can conclude that IRrate does not affect the tracking performance, when with the same EXrate.

4.2. The Performance with Different Models. Next, we compare the model developed here with other dynamics model, such as CV model [12], CA model [12], Singer model [13], current model [8], and IMM [14]. We set the process noise covariance as $Q=1$ for the $\mathrm{CV}$ and CA model and $\sigma_{w}^{2}=1$ and $\alpha=1 / 20$ for Singer model. Because the current model is very sensitive to the priori parameters, we give several system parameters such as $\alpha=1 / 30, \alpha_{\max }=3$ (current model I), $\alpha=$ $1 / 20, \alpha_{\max }=30$ (current model II), and $\alpha=1 / 20, \alpha_{\max }=3$ (current model III). After 100 Monte Carlo simulation runs, $\mathrm{RMSE}_{2 \mathrm{D}}$ are calculated. For different trajectory with different EXrate and IRrate, the estimation results are shown in Figures $7(\mathrm{a})-7(\mathrm{f})$, where in order to show clearly, we use the black " $\mathrm{O}$ " to describe the actual trajectory at the sampling time in Figures 7(e) and 7(f).

Table 2 and Figure 8 show RMSE $_{2 \mathrm{D}}$ under the different IRrate and EXrate. We can see that the model here can get the better estimation performance than CV, CA, Singer model, current model, and IMM for almost all EXrate and IRrate. We also note that the current model needs the right parameter, or else the performance will become worse.

We note that in Figure 7(f), the tracking error of the developed model is larger than current models II, III, and IMM, even CA. We find that there is a big estimation error at 5 th second. The reason is that there are not enough data gotten to update the parameter at $K_{0}=4$. Therefore, the estimation error is bigger. But we also note that the estimation error declined quickly, so the developed model has a strong advantage for the long trajectory tracking comparing the other models.

Another fact we also noticed is that though IRrate almost does not affect the tracking performance, it is obvious that low EXrate can decline the tracking performance. This is because the lower EXrate means less measured data gotten and less useful information that can be provided; therefore, the estimate is more inaccurate.

As to the sampling interval th $\mathrm{h}_{i}$, the lower EXrate means larger $t_{i}$. If the sampling interval th ${ }_{i}$ is large enough to break
Shannon Sampling Theorem, the estimation performance will decline.

4.3. The Estimation of Video Target. At last, we use the developed method to track a target in real scene. In order to decrease the calculation cost, we select some frames from the video according to the characteristics of the movement. That is, if we find that the target is stationary or moves slowly, then we discard these frames. We use a threshold to test whether a target makes a big maneuver or not. Obviously, a large threshold can make the calculation cost lower, but lower EXrate will make the performance decrease too.

So the threshold should be carefully selected to balance the calculation cost and performance. Here, we select 95 frames from 245 frames; EXrate and IRrate are 38.77\% and 0.1367, respectively. Figure 9 gives the tracking results of number 1, 27, 40,65, 74, 97, 128, 129, 158, 181, 189, and 226 frames in the video. The estimation of target is marked by "black" dot. The estimation covariance of $\mathrm{RMSE}_{2 \mathrm{D}}$ as $1.034 \mathrm{~mm}$ is obtained (the tracking area is $300 * 300 \mathrm{~mm}^{2}$ ).

\section{Conclusions}

The main contribution of this paper is to model the realtime system dynamics at the random sampling points. (1) By calculating the matrix exponential with inverse Laplace transform, the irregular sampling interval is transformed to time-varying parameters matrix of the system. (2) Based on the statistics relation between the autocorrelation function and the covariance of Markov random processing, the system model with online parameter is developed. The proof and the experimental results show that the developed method can get good tracking performance.

As an example, the developed method is used for the video tracking problem. According to the motion characteristics of the target, some frames are selected for the tracking purpose. The tracking results show that good tracking performance is obtained by a smaller amount of calculation.

\section{Disclosure}

The authors declare that they have no financial or personal relationships with other people or organizations that can inappropriately influence their work and there is no professional or other personal interest of any nature in any product, service, and/or company that could be construed as influencing the position presented in this paper.

\section{Conflict of Interests}

The authors declare that there is no conflict of interests regarding the publication of this paper. 


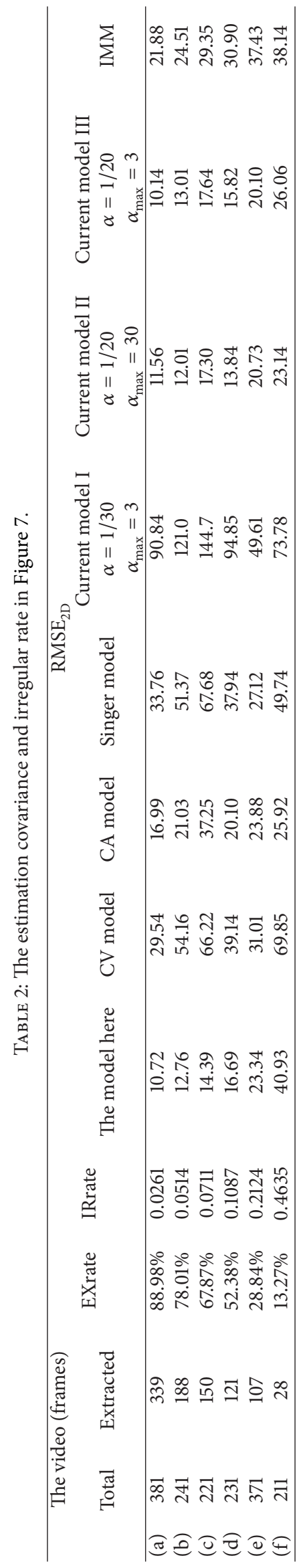




\section{Acknowledgments}

This work is partially supported by NSFC under Grant nos. 61273002 and 60971119 and the Importation and Development of High-Caliber Talents Project of Beijing Municipal Institutions no. CIT\&TCD201304025.

\section{References}

[1] S. Srinivasan and H. Ranganathan, "RFID sensor networkbased automation system for monitoring and tracking of sandalwood trees," International Journal of Computational Science and Engineering, vol. 8, no. 2, pp. 154-161, 2013.

[2] S. Yin, S. X. Ding, A. H. A. Sari, and H. Hao, "Data-driven monitoring for stochastic systems and its application on batch process," International Journal of Systems Science, vol. 44, no. 7, pp. 1366-1376, 2013.

[3] F. Dornaika and F. Chakik, "Efficient object detection and tracking in video sequences," Journal of the Optical Society of America A, vol. 29, no. 6, pp. 928-935, 2012.

[4] J. Xue-Bo, D. Jing-Jing, and B. Jia, "Fast tracking for video target tracking," Applied Mechanics and Materials, vol. 303-306, pp. 2245-2248, 2013.

[5] H. Zhang, M. V. Basin, and M. Skliar, "Itô-Volterra optimal state estimation with continuous, multirate, randomly sampled, and delayed measurements," Institute of Electrical and Electronics Engineers, vol. 52, no. 3, pp. 401-416, 2007.

[6] J. Xue-Bo, D. Jing-Jing, and B. Jia, "Target tracking of a linear time invariant system under irregular sampling," International Journal of Advanced Robotic Systems, vol. 9, no. 11, pp. 1-12, 2012.

[7] S. Vasuhi, V. Vaidehi, and T. Rincy, "IMM estimator for maneuvering target tracking with Improved current statistical model," in Proceedings of the International Conference on Recent Trends in Information Technology (ICRTIT '11), pp. 286-290, June 2011.

[8] W.-S. Liu, Y.-A. Li, and L. Cui, "Adaptive strong tracking algorithm for maneuvering targets based on current statistical model," Systems Engineering and Electronics, vol. 33, no. 9, pp. 1937-1940, 2011.

[9] W. Wang and H.-L. Hou, "An improved current statistical model for maneuvering target tracking," in Proceedings of the 4th IEEE Conference on Industrial Electronics and Applications (ICIEA '09), pp. 4017-4020, May 2009.

[10] H. Li and C. Li, "Missile-borne radar data filtering algorithm based on the "current" statistical model," Advanced Materials Research, vol. 433-440, pp. 6965-6973, 2012.

[11] Y.-L. Liu and X.-H. Gu, "Current statistical model tracking algorithm based on improved auxiliary particle filter," Systems Engineering and Electronics, vol. 32, no. 6, pp. 1206-1209, 2010.

[12] X. R. Li and V. P. Jilkov, "Survey of maneuvering target tracking. Part I: dynamic models," IEEE Transactions on Aerospace and Electronic Systems, vol. 39, no. 4, pp. 1333-1364, 2003.

[13] X. Chen, Y. Pang, Y. Li, and D. Li, "AUV sensor fault diagnosis based on STF-Singer model," Chinese Journal of Scientific Instrument, vol. 31, no. 7, pp. 1502-1508, 2010.

[14] T.-J. Ho, "A switched IMM-extended Viterbi estimator-based algorithm for maneuvering target tracking," Automatica, vol. 47, no. 1, pp. 92-98, 2011.

[15] R. W. Osborne and W. D. Blair, "Update to the hybrid conditional averaging performance prediction of the IMM algorithm," IEEE Transactions on Aerospace and Electronic Systems, vol. 47, no. 4, pp. 2967-2974, 2011.
[16] X. Bian, X. R. Li, H. Chen, D. Gan, and J. Qiu, "Joint estimation of state and parameter with synchrophasors-Part I: state tracking," IEEE Transactions on Power Systems, vol. 26, no. 3, pp. 1196-1208, 2011.

[17] X. Bian, X. R. Li, H. Chen, D. Gan, and J. Qiu, "Joint estimation of state and parameter with synchrophasors-Part II: parameter tracking," IEEE Transactions on Power Systems, vol. 26, no. 3, pp. 1209-1220, 2011.

[18] E. Wensink and W. J. Dijkhof, "On finite sample statistics for Yule-Walker estimates," Institute of Electrical and Electronics Engineers, vol. 49, no. 2, pp. 509-516, 2003. 


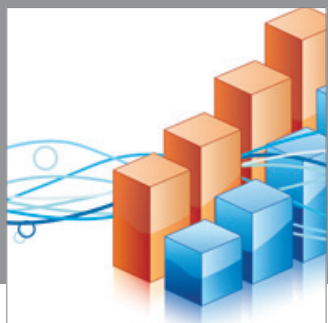

Advances in

Operations Research

mansans

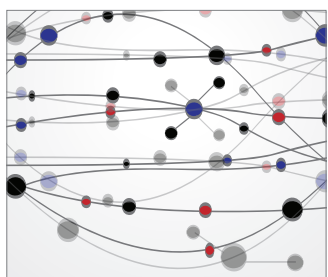

The Scientific World Journal
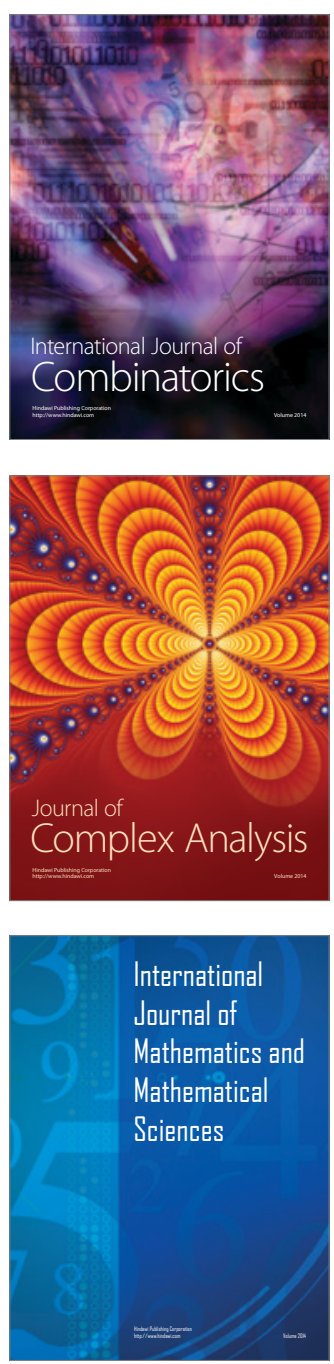
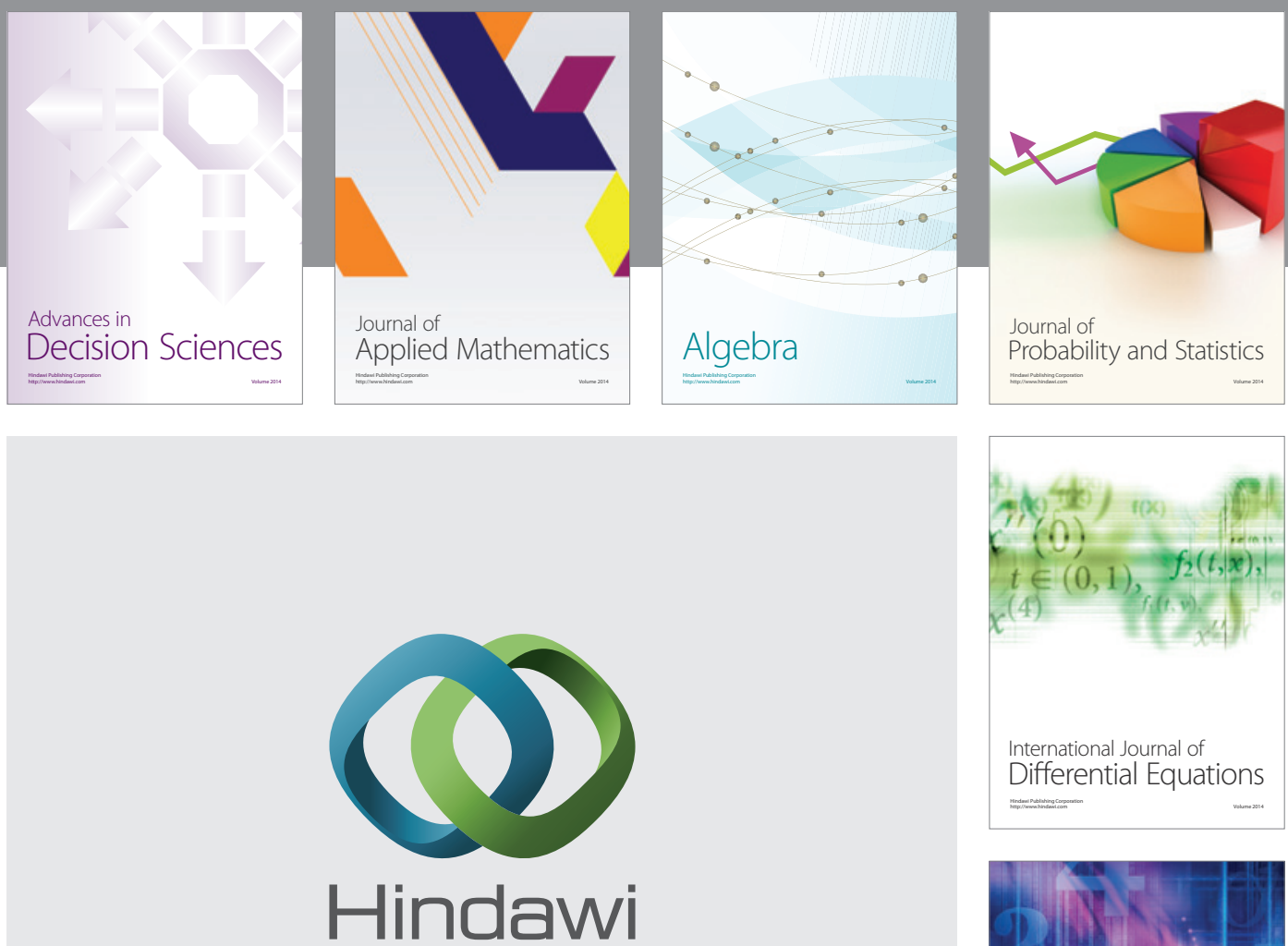

Submit your manuscripts at http://www.hindawi.com
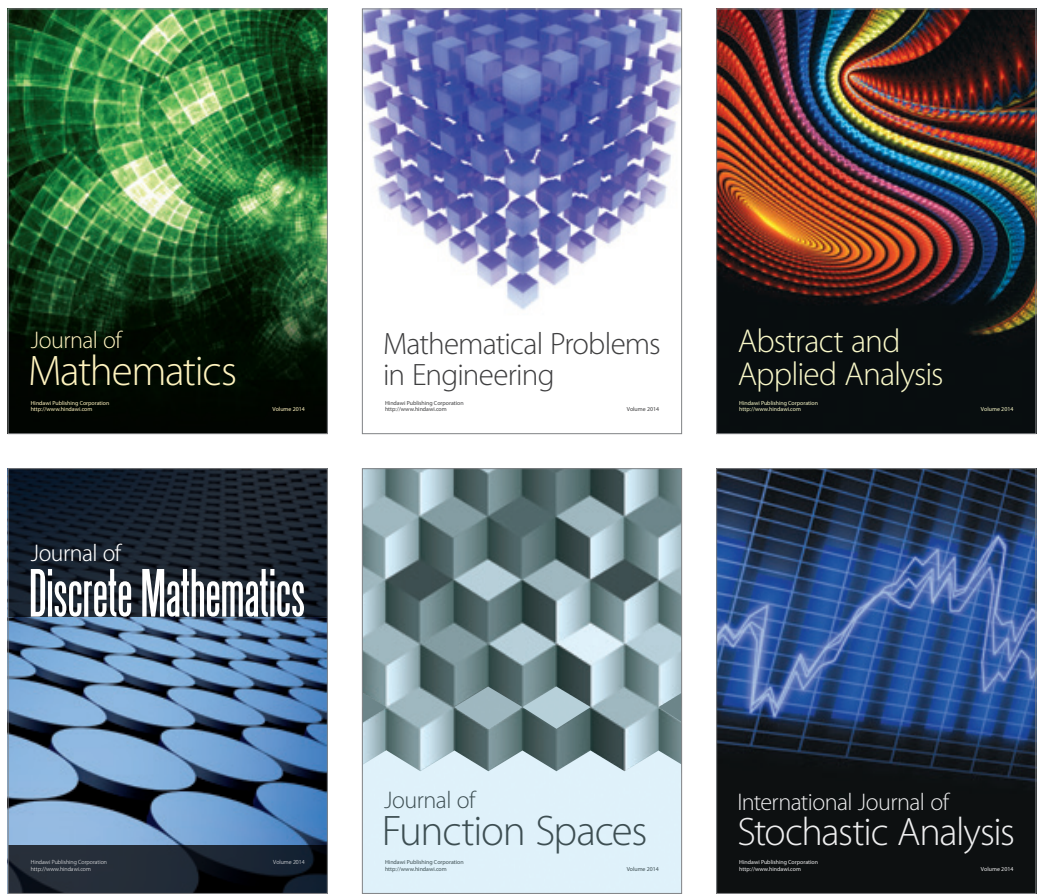

Journal of

Function Spaces

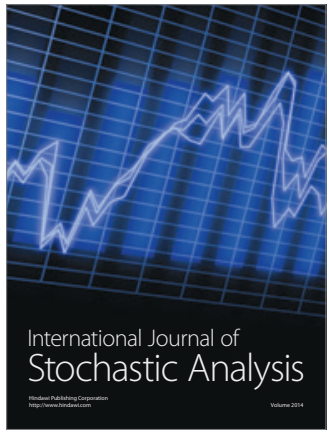

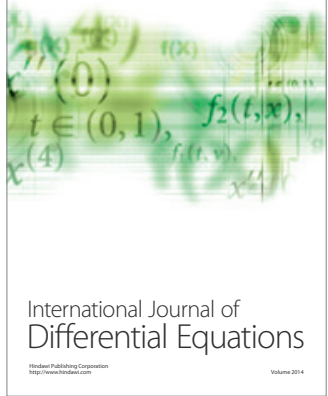
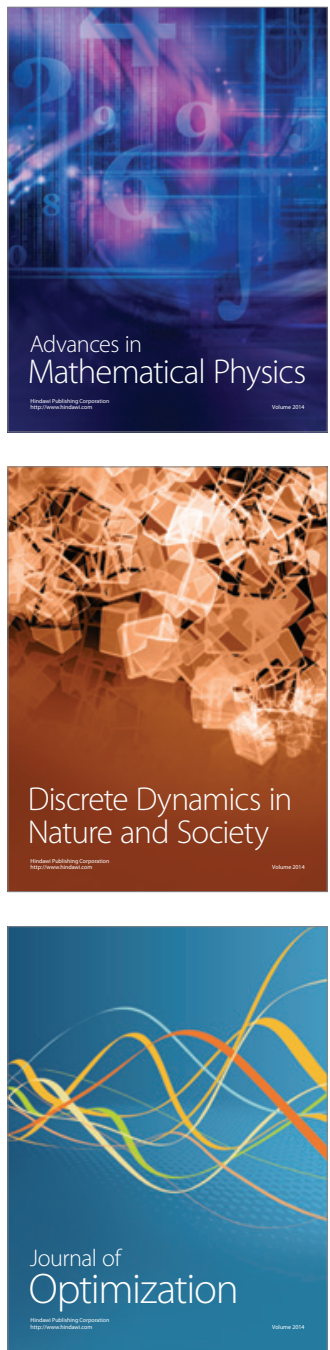